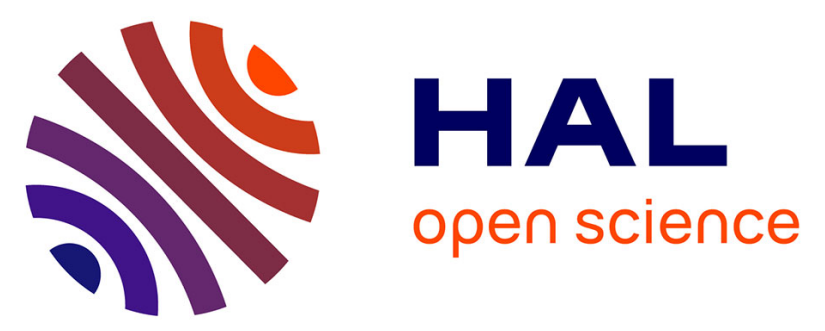

\title{
Interventions des pharmaciens sur les prescriptions ambulatoires dans le cadre de l'activité de rétrocession d'un centre hospitalier universitaire
}

\author{
Mathieu Chappuy, Stephan Garcia, Anne-Claire Uhres, Audrey
}

Janoly-Dumenil, Julia Dessault, Valérie Chamouard, Valentine Breant, Gilles

Leboucher, Christine Pivot, Isabelle Carpentier

\section{To cite this version:}

Mathieu Chappuy, Stephan Garcia, Anne-Claire Uhres, Audrey Janoly-Dumenil, Julia Dessault, et al.. Interventions des pharmaciens sur les prescriptions ambulatoires dans le cadre de l'activité de rétrocession d'un centre hospitalier universitaire. Annales Pharmaceutiques Françaises, 2015, 10.1016/j.pharma.2014.11.001. 10.1016/j.pharma.2014.11.001 . hal-01107608

\section{HAL Id: hal-01107608 \\ https://hcl.hal.science/hal-01107608}

Submitted on 21 Jan 2015

HAL is a multi-disciplinary open access archive for the deposit and dissemination of scientific research documents, whether they are published or not. The documents may come from teaching and research institutions in France or abroad, or from public or private research centers.
L'archive ouverte pluridisciplinaire HAL, est destinée au dépôt et à la diffusion de documents scientifiques de niveau recherche, publiés ou non, émanant des établissements d'enseignement et de recherche français ou étrangers, des laboratoires publics ou privés. 
Interventions des pharmaciens sur les prescriptions ambulatoires dans le cadre de l'activité de rétrocession d'un Centre Hospitalier Universitaire.

Pharmacist's interventions on outpatient prescriptions in a University Hospital drugs sales service.

Mathieu Chappuy ${ }^{1 *}$, Stéphan Garcia ${ }^{2}$, Anne Claire Uhres ${ }^{3}$, Audrey Janoly-Dumenil ${ }^{1}$, Julia Dessault $^{2}$, Valérie Chamouard ${ }^{4}$, Valentine Bréant ${ }^{4}$, Gilles Leboucher ${ }^{3}$, Christine Pivot $^{1}$, Isabelle Carpentier ${ }^{2}$.

1: Service Pharmaceutique, Groupement Hospitalier Edouard Herriot, 5 Place d'Arsonval 69003 Lyon, France.

2 : Pharmacie Centrale des Hospices Civils de Lyon, 57 rue Francisque Darcieux 69230 Saint Genis Laval, France.

3: Service Pharmaceutique, Groupement Hospitalier Nord, 103 Grande Rue de la Croix Rousse 69004 Lyon, France.

4 : Service Pharmaceutique, Groupement Hospitalier Est, 28 Avenue Doyen Lépine 69500 Bron, France.

* : téléphone : 04721109 98, fax : 04721104 11, e-mail : mathieu.chappuy@chu-lyon.fr 


\section{Résumé :}

Pour des raisons de santé publique, certains médicaments ne peuvent être dispensés aux patients ambulatoires que par les pharmacies à usage intérieur dans le cadre de la rétrocession. Cette activité s'exerce dans un contexte de risque spécifique lié aux organisations, aux patients et/ou aux médicaments. L'analyse systématique des prescriptions par un pharmacien concourt à la sécurisation des traitements dispensés. L'objectif de cet article est de présenter les principaux problèmes médicamenteux rencontrés lors de l'analyse des prescriptions pour les patients ambulatoires et les interventions pharmaceutiques en découlant au sein de trois unités de rétrocession d'un Centre Hospitalier Universitaire.

Méthodes : De janvier à décembre 2013, les problèmes médicamenteux détectés ont été enregistrés de manière systématique et prospective.

Résultats : Sur les 22279 prescriptions analysées, 247 interventions pharmaceutiques $(1,1 \%)$ ont été formulées avec notamment $27,6 \%$ des problèmes liés aux posologies, $15,4 \%$ de nonconformité aux référentiels, $6,9 \%$ des contre-indications. Les anti-infectieux $(43,7 \%)$ et les anticancéreux $(17,4 \%)$ sont les classes médicamenteuses les plus impliquées. Le taux d'acceptation global est de $81,8 \%$.

Conclusion : Ces résultats montrent l'intérêt de l'analyse des prescriptions ambulatoires lors de la dispensation et la nécessité de disposer de l'ensemble des prescriptions du patient des éléments cliniques, biologiques et de développer l'interprofessionnalité. La mise en œuvre d'une plateforme d'échanges de données dématérialisées entre les professionnels, intégrant notamment les données issues du dossier pharmaceutique du patient, contribuerait à l'amélioration de la prise en charge médicamenteuse du patient. 


\section{Abstract:}

For public health reasons, some drugs are only available in hospital drugs sales service. This activity takes place in a specific risk context of organization, patients and/or drugs. A systematic prescriptions analysis by pharmacist contributes to securise treatment dispensed.

The aim of this paper is to present the main drugs problems in the analysis of outpatient prescriptions and pharmaceutical interventions in three units of hospital drugs sales service belong to university hospital.

Methods: Throughout the year 2013, drug problems detected were recorded prospectively and systematically.

Results: Of the 22279 prescriptions analyzed 247 pharmaceutical interventions (1.1\%) were detected including $27.6 \%$ of problems concerns the dosages, $15.4 \%$ the unconformity, $6.9 \%$ contraindications. Regarding ATC drugs classes, we found $43.7 \%$ for antiinfectives and $17.4 \%$ for antineoplatics. The overall acceptance rate is $81.8 \%$.

Conclusion: These results show the importance of the analysis of outpatient prescriptions before dispensing and the need to have all prescriptions, clinical and biological elements and to develop interprofessionality.

The implementation of a platform for dematerialized data exchanges between professionals, including data from the pharmaceutical patient record should contribute to improving drug management of the patient. 
Mots clés : interventions pharmaceutiques, prescriptions, rétrocession, problème médicamenteux, hôpital-ville.

Keys words: pharmaceutical interventions, prescriptions analysis, hospital drugs sales service, medications errors, hospital-city. 


\section{Introduction :}

En France, certains médicaments prescrits aux patients ambulatoires ne peuvent être dispensés que par les pharmacies hospitalières autorisées, dans le cadre de la rétrocession. Les critères qui prévalent pour l'inscription des médicaments sur la liste de rétrocession doivent répondre à l'intérêt des malades : contraintes particulières de distribution, de dispensation ou d'administration (exemple : anti-rétroviraux); sécurité de l'approvisionnement (exemple : facteurs de la coagulation); nécessité d'effectuer un suivi de la prescription ou de la dispensation [1].

Cette mission optionnelle des Pharmacies à Usage Intérieur (PUI) nécessite une autorisation de l'Agence Régionale de Santé. Plusieurs catégories de médicaments sont concernées :

○ Ceux inscrits par arrêté sur la liste de rétrocession (article L5126-4 du Code de la Santé Publique), soit au titre de l'Autorisation de Mise sur le Marché (AMM), soit au titre d'une Autorisation Temporaire d'Utilisation (ATU) de cohorte ;

○ Ceux sous ATU nominative, non réservés à l'usage hospitalier ;

○ Certains médicaments importés (hors importations parallèles), notamment pour pallier les ruptures de stock en ville ;

○ Des préparations magistrales et hospitalières ;

- Des médicaments dispensés dans le cadre de la prise en charge de la douleur chronique rebelle et des soins palliatifs, pour assurer la continuité des soins.

L'activité de rétrocession est une activité spécifique complexe à plusieurs titres. D’une part, il s'agit d'une activité exercée à l'interface entre une organisation hospitalière et celle de ville. Ceci multiplie les intervenants (hospitaliers et/ou libéraux) et le risque d'incohérence des traitements du fait de circuits multiples de prescription. Pour certains patients, le parcours de soins est complexe, alternant épisodes d'hospitalisation, voire hospitalisation à domicile, domicile, constituant autant de facteurs de risque de rupture dans la chaîne de soins. Cette 
rupture est d'autant plus problématique que ces patients présentent des pathologies rares et/ou graves et qu'une interruption de traitement peut être rapidement délétère. D'un point de vue pharmaceutique, les rétrocessions sont complexes, car elles s'adressent à des patients souvent poly-médicamentés car poly-pathologiques. D'autre part, les médicaments dispensés peuvent être qualifiés de médicaments à risque. A ce titre, certaines spécialités dispensées en rétrocession font l'objet d'un plan de gestion des risques (exemples: thalidomide, lénalidomide). Ce risque est majoré lorsqu'il s'agit de spécialités sous statut d'ATU (parfois peu connues et peu documentées) et nécessitant souvent une surveillance renforcée et le respect des protocoles d'utilisation thérapeutique (PUT).

En France, les médicaments rétrocédés représentaient en 2011 une dépense de plus d'1,3 milliards d'euros soit environ $7 \%$ des 19 milliards d'euros remboursés par l'assurance maladie (régime général) au titre des médicaments [2], ou encore 1\% des dépenses globales du régime général de la sécurité sociale. Les médicaments rétrocédés par les PUI des Hospices Civils de Lyon (HCL), deuxième Centre Hospitalo-Universitaire de France, représentaient en 2013 un montant de 42,2 millions d'euros.

Afin de concourir à la qualité et à la sécurité des traitements dispensés aux patients ambulatoires, l'ensemble des prescriptions médicales est analysé par un pharmacien, en lien avec les prescriptions de ville quand elles sont disponibles et donnent lieu à des Interventions Pharmaceutiques (IP) auprès des prescripteurs [3]. Cette analyse est toutefois variable par son niveau [4]. En effet, au regard des recommandations de la Société Française de Pharmacie Clinique (SFPC), le pharmacien réalise une analyse de niveau 1 lorsqu'il fait une revue de la prescription chez un patient connu. Elle sera de niveau 2 lorsque nous pouvons vérifier les posologies en lien avec les résultats biologiques ou des événements traceurs (exemple : le patient relate un événement indésirable). Le niveau 3 implique le respect des objectifs thérapeutiques et de l'observance sans oublier les conseils et l'éducation thérapeutique. 
Cependant, outre l'analyse pharmaco-thérapeutique, il convient de prendre en compte le cadre réglementaire strict qui concourt à la sécurisation de la prise en charge médicamenteuse et conditionne le remboursement par l'Assurance Maladie (appartenance des spécialités à la liste prévue à l'article L.5126-4 du Code de la Santé Publique, habilitation des prescripteurs, respect des PUT, suivi des indications dans le cadre du dispositif AMM post ATU, durée de validité des ATU, durée maximale de la prescription, ...).

Il existe de nombreuses études concernant les problèmes médicamenteux détectés par les pharmaciens sur les prescriptions hospitalières [3, 5-10] et qui évaluent l'impact clinique [7-9] ou économique [10] mais de telles études spécifiques à l'activité de rétrocession n'ont pas été publiées en France.

L'objectif de ce travail est de décrire d'une part les principaux problèmes médicamenteux détectés lors de l'analyse des prescriptions et d'autre part présenter les interventions pharmaceutiques formulées par les pharmaciens au sein de trois sites de rétrocession des HCL. 


\section{Matériels et Méthodes :}

Aux HCL, quatre sites répartis sur l'ensemble de l'agglomération lyonnaise ont assuré la prise en charge de 5538 patients en 2013. Ces sites, situés au sein des principaux groupements hospitaliers, affichent pour certains des consultations spécialisées (infectiologie, onco-hématologie, hémophilie, pneumo-cardiologie, maladies métaboliques, pédiatrie,...).

Sous l'impulsion du Groupe de travail Pharmacie Clinique du pôle d'activité pharmaceutique des HCL, un sous-groupe dédié à l'activité ambulatoire a été créé en 2008, dont l'objectif était d'améliorer la sécurisation de la prise en charge médicamenteuse du patient ambulatoire au sein d'un site de rétrocession des HCL.

Une étude prospective a été conduite du $1^{\text {er }}$ janvier au 31 décembre 2013 sur trois sites de rétrocession dotés chacun d'un pharmacien, un étudiant en $5^{\text {ème }}$ année hospitalo-universitaire et deux préparateurs en pharmacie hospitalière. Le $4^{\text {ème }}$ site, par manque de moyens, n'a pas pu enregistrer ses interventions pharmaceutiques. L'analyse des prescriptions par le professionnel de santé avec double contrôle du pharmacien était réalisée lors de chaque dispensation, soit en temps réel si le patient se présentait directement, soit par anticipation si nous étions prévenus (prescriptions faxées), y compris en cas de renouvellement. Dans notre pratique, le niveau 1 concerne les patients non suivis aux HCL, pour lesquels nous n'avons pas accès au dossier médical. Les informations sont parcellaires ; il est difficile de recueillir l'ensemble des prescriptions et données médicales, d'autant que le lien institutionnel est absent. En revanche, pour les patients suivis aux HCL, notre analyse est de niveau 2 voire 3. En effet, l'accès au dossier médical commun informatisé est facilité, comprenant l'intégralité des données biologiques, les comptes rendus d'hospitalisation ou de consultation. Néanmoins, dans tous les cas, l'une des difficultés reste l'accès à une information exhaustive, puisque la consultation du dossier pharmaceutique du patient créé par une pharmacie d'officine n'est pas encore possible et que, sans le concours du patient, l'accès à l'intégralité des prescriptions est 
impossible. En effet, selon les cas, nous pouvons disposer de l'intégralité du traitement (ville/hôpital) sur le même support, ou à l'inverse, uniquement les spécialités rétrocédables.

Si un problème médicamenteux était repéré, une intervention était réalisée directement auprès du clinicien, par téléphone ou par mail, du fait de l'éloignement géographique des unités de soins et tracée immédiatement sur la prescription médicale dont une copie est conservée en vue du recueil.

Dans le cadre de la standardisation du recueil réalisée aux HCL, les problèmes médicamenteux rencontrés et les solutions proposées ont été saisis dans la base ACT-IP® développée sous l'égide de la SFPC [11,12], puis analysés à l'aide du logiciel Excel® (Office 2010, Microsoft Corporation, USA).

Un précédent travail a montré que des difficultés de cotation sont rencontrées dans $11 \%$ des cas (exemple : «non-conformité de la prescription médicale ») du fait d'une inadaptation partielle de la base ACT-IP® à l'activité de rétrocession, entraînant une éventuelle sousnotification et a conduit à une adaptation de la grille de recueil [13].

De ce fait, des mots-clés ont été ajoutés en commentaire. Par exemple, pour préciser l'item «non-conformité aux référentiels et contre-indication » nous avons identifié : « le prescripteur n'est pas autorisé », « l'ATU n'est pas valide » ou «non-respect du PUT ».

De même, l'item «non-conformité du choix du médicament au livret thérapeutique »a été utilisé pour les IP réalisées lorsque le médicament prescrit n'était pas inscrit sur la liste de rétrocession (disponible en officine ou réservé à l'usage hospitalier). L'item « un même principe actif est prescrit plusieurs fois sur l'ordonnance »a été utilisé en cas de redondance du principe actif sur plusieurs prescriptions (ville/hôpital).

L'item «médicament non prescrit après un transfert » a pu être utilisé dans des situations de ruptures liées à des problèmes d'approvisionnement de médicament en ville, lors de la 
suppléance par la PUI avec un médicament d'importation autorisé par l'Agence Nationale de Sécurité du Médicament. 


\section{Résultats :}

Au total 22279 prescriptions comportant 37411 lignes de médicaments ont été analysées, correspondant à la prise en charge de 5538 patients. Dans 49,5\% des cas, il s'agissait de prescriptions initiales $(\mathrm{n}=11017)$. Ces prescriptions provenaient de médecins exerçant soit aux HCL, soit dans d'autres établissements de santé (publics ou privés) soit en libéral (généralistes ou spécialistes). Les prescriptions étaient établies pour une durée moyenne de 3,1 mois [1 mois -12 mois].

Le sexe ratio homme/femme des patients était de 1,3. L'âge moyen était de 52 ans [1 mois 93 ans]. Les prescripteurs concernés par les IP étaient à $85 \%$ des seniors, $15 \%$ des internes en médecine. Ils travaillent à $72 \%$ au sein des HCL. La transmission a été faite à 91,1\% par téléphone (accompagnée par un mail dans $8,4 \%$ des cas), dans 7,7 \% par mail uniquement et dans $1,2 \%$ oralement directement au patient.

Les caractéristiques des problèmes médicamenteux rencontrés et leur fréquence sont présentées dans le tableau 1. Les problèmes récurrents sont détaillés ci-après. Ils ont donné lieu à $247 \mathrm{IP}$, soit 1,1\% des prescriptions analysées (tableau 2).

\section{$\circ$ Surdosages $/$ sous-dosages $(\mathbf{n}=\mathbf{7 1})$}

Près d'un tiers des problèmes médicamenteux relevés concernaient les posologies. Dans $15 \%$ des cas, elles étaient inférieures aux recommandations ou à celles habituellement prescrites, sans aucune information délivrée au patient ni renseignée dans le dossier médical. Dans $13,8 \%$ des cas, les posologies étaient supra-thérapeutiques. La variation enregistrée était le plus souvent d'un facteur 2. Lorsqu'il s'agissait d'un sous-dosage, la réduction de posologie moyenne était de $47 \%$ [2-90\%] et pour les surdosages, la variation moyenne de posologie était de $94 \%$ [5- $400 \%]$. Dans 54,9\% ( $=39 / 71)$, le problème était détecté du fait d'une discordance entre le traitement prescrit et l'historique médicamenteux du patient. 
Les deux principales classes médicamenteuses concernées étaient les anti-infectieux (56,3\%) et les anticancéreux $(16,9 \%)$.

\section{$\bigcirc$ Non-conformité aux référentiels et contre-indications $(\mathbf{n}=\mathbf{5 8})$}

Des problèmes de non-conformité aux référentiels ou contre-indication (tous types) étaient relevés dans 23,5\% des cas. On retrouve (tableau 3) la non-conformité aux PUT ou la non validité des ATU (6,9 \% des IP totales) mais aussi la problématique des prescripteurs non habilités $(6,5 \%$ des IP totales) lorsque des médicaments requièrent une spécialisation médicale (exemple des anticancéreux, des médicaments de l'hypertension artérielle pulmonaire (HTAP)) ou lorsqu'un médecin libéral renouvelle un traitement nécessitant une prescription hospitalière. A noter que 3,2 \% des problèmes concernaient des médicaments non rétrocédables (exemples : l'amoxicilline injectable, l'imipenem/cilastatine) pour lesquels il faut éventuellement proposer une alternative thérapeutique disponible pour l'usage ambulatoire ou encore une prise en charge dans le cadre d'une hospitalisation à domicile ou d'un réseau de soins palliatifs par exemple. Concernant les contre-indications $(0,8 \%)$, nous retrouvons la prescription d'une spécialité d'importation (Antiscabiosum ${ }^{\circledR}$ ) pour pallier la rupture française (Ascabiol ${ }^{\circledR}$ ) qui est prescrite chez des nourrissons atteints de gale alors que l'AMM la contre-indique en dessous de un an.

\section{○ Interactions}

Les problèmes rencontrés concernaient les interactions médicamenteuses dans $12,5 \%$ des cas $(\mathrm{n}=31)$, avec un niveau d'associations contre-indiquées ou déconseillées dans $10,9 \%$ des cas $(n=27)$. Les principales interactions détectées impliquaient le linézolide avec des inhibiteurs de la recapture de la sérotonine ou le tramadol (risque d'apparition d'un syndrome 
sérotoninergique) et les antifongiques (posaconazole, voriconazole, kétoconazole) avec des médicaments métabolisés par le cytochrome P450 3A4 (par exemple l'atorvastatine).

\section{○ Voie et/ou administration inappropriée $(n=33)$}

Les problèmes relatifs à l'item « voie et/ou administration inappropriée » regroupent aussi bien un libellé incomplet qu'une méthode d'administration non adéquate, un plan de prise non optimal ou un mauvais choix de forme galénique, et représentaient $13,4 \%$ des interventions $(n=33)$.

On retrouvait toutes les classes médicamenteuses, que ce soit les anticancéreux (exemple : le régorafénib prescrit en deux prises par jour au lieu d'une prise dans l'autorisation de mise sur le marché), les antibiotiques (exemples : l'ertapénem en intramusculaire, le voriconazole prescrit aux repas) mais aussi des médicaments non rétrocédables (exemple : le paracétamol en comprimés effervescents contenant une quantité non négligeable de sodium chez des patients insuffisants rénaux et hypertendus [14]).

\section{$\bigcirc$ Indication non traitée $(\mathbf{n}=19)$}

Ce problème correspondait principalement aux médicaments en rupture de stock en ville $(n=14)$ pour lequels une alternative thérapeutique différente et nécessitant une nouvelle prescription était possible (exemple : Oncotice ${ }^{\circledR}$ en remplacement d'Immucyst $^{\circledR}$ ) et que nous codions par défaut comme médicament non prescrit après transfert. En effet, dans ces cas, la substitution par le pharmacien n'est pas possible et cette situation génère, en l'absence d'intervention opportune auprès du prescripteur, une rupture potentielle dans la chaîne des soins. 
Par ailleurs, cet item englobait dans une moindre mesure $(n=5)$ l'absence thérapeutique pour indication médicale validée (exemple : la non prescription de dexaméthasone chez un patient sous protocole lénalidomide/dexaméthasone dans l'indication d'un myélome multiple).

\section{○ Caractéristiques des IP}

\section{$\circ \quad$ Classe ATC (tableau 4)}

Près de la moitié des problèmes $(43,7 \%)(\mathrm{n}=108)$ concernaient la classe des anti-infectieux à usage systémique. La classe des antinéoplasiques et agents immuno-modulateurs représentait 17,4\% $(\mathrm{n}=43)$. A noter que les interventions concernant le sildénafil dans le cadre de 1'HTAP sont répertoriées dans la classe «Système génito-urinaire et hormones sexuelles ». A lui seul, le sildénafil représentait 4,5\% des IP effectuées.

\section{○ Corrélation entre problème détecté et IP (tableau 2)}

Il existe une corrélation entre le problème détecté et l'intervention proposée. Ainsi les problèmes de sur- ou sous-dosage donnaient lieu à une proposition d'adaptation posologique $(29,1 \%$ des cas, $\mathrm{n}=72)$, les problèmes de non-conformité aux référentiels à l'ajout d'une prescription nouvelle $(20,6 \%$ des cas, $\mathrm{n}=51)$ ou encore à une substitution ou un échange dans $11,7 \%$ des cas $(n=29)$.

Les IP proposées ont été acceptées dans 81,8\% et refusées dans 10,1\%. Dans 8,1\% des cas, le devenir de l'intervention n'est pas documenté. 


\section{Discussion:}

Il s'agit de la première étude décrivant les problèmes pharmaco-thérapeutiques rencontrés lors de l'analyse des prescriptions ambulatoires dans le cadre de la rétrocession en France. Une étude prospective large portant sur les prescriptions d'hospitalisation et ambulatoire a été réalisée aux HCL en 2007-2008 [3], sans analyse par secteur.

\section{$\bigcirc$ Taux d'interventions}

Notre étude montre un taux d'interventions pharmaceutiques faible, par rapport aux taux communément rapportés en hospitalisation [3, 5-10]. Plusieurs facteurs peuvent expliquer ce résultat :

- la grille de recueil développée par la SFPC n'est pas totalement adaptée à l'exercice ambulatoire [13]. En effet, malgré les modifications apportées, il subsiste probablement une sous-notification liée au professionnel de santé plus ou moins sensibilisé, à la charge de travail ;

○ La majorité des prescriptions analysées $(50,5 \%)$ correspond à un renouvellement de traitement. Or, si un problème médicamenteux est détecté à la première dispensation, il sera documenté et ne donnera lieu qu'à une seule intervention. Lors des renouvellements de traitements, le problème ne devrait donc pas être récurrent. Contrairement à l'hospitalisation, cette spécificité entraîne une augmentation de notre dénominateur et donc un taux d'intervention plus faible. Néanmoins, tous les renouvellements sont analysés car certaines IP sont faites lors des renouvellements (exemples: modification de la posologie de l'antibiotique suite aux taux résiduels, prescription pour une durée supérieure à la réglementation comme par exemple le thalidomide prescrit sur plus de 12 semaines et qui n'a pas 
donné lieu à une IP auparavant considérant que le patient serait revu avant par son médecin et qu'une nouvelle prescription serait faite).

○ Nous ne disposons pas systématiquement de l'ensemble des traitements médicamenteux des patients, en particulier lorsqu'ils ne sont pas suivis aux HCL. L'absence d'accès aux dossiers médicaux (traitements chroniques, indications) et aux résultats des analyses de biologie médicale rend l'analyse pharmaceutique plus succincte et des problèmes médicamenteux peuvent ne pas être détectés.

\section{○ Problèmes rencontrés}

\section{○ Erreurs de posologie et interactions médicamenteuses}

Le premier motif d'intervention est lié à un problème de posologie. Or, dans plus de la moitié des cas, ces erreurs sont relevées du fait d'une discordance entre le traitement prescrit et l'historique médicamenteux du patient (exemple: le renouvellement par un médecin remplaçant sans tenir compte d'une adaptation de dose). Les taux d'acceptation de l'ordre de $95 \%$ relevés dans ce cas montrent qu'il s'agit bien d'erreurs de prescription avérées et non d'une modification intentionnelle du traitement par le prescripteur. Ce motif se retrouve également en hospitalisation $[3,15]$ avec néanmoins un taux d'acceptation plus faible $(54 \%$, 67-70 \%) qui s'explique par la nature des médicaments concernés (hypnotique à pleine dose chez le sujet âgé, dose cumulée de paracétamol présente dans diverses spécialités).

De même, les interactions médicamenteuses détectées impliquent régulièrement un médicament rétrocédé et un médicament de ville prescrit dans le cadre d'un traitement chronique (exemple : dyslipidémie, douleur, prostate).

La multiplicité des prescripteurs pour un même patient, au sein d'un même service d'une part, entre la ville et l'hôpital d'autre part, concourt probablement à accentuer ces problèmes. 
Il est difficile de disposer de l'intégralité des prescriptions de sortie ; en effet, le plus souvent, les prescriptions pour les traitements de ville et rétrocédés ne sont pas rédigées sur un même support. Ainsi, le lien avec les traitements chroniques gérés en ville est parfois difficile, d'autant que le patient n'est pas toujours présent (aidant, ambulancier). Ces exemples soulignent l'importance de la conciliation des traitements médicamenteux du patient (hospitaliers et ambulatoires), qui est réalisée à chaque rétrocession. Le Dossier Pharmaceutique (DP) partagé entre ville et hôpital, ainsi que la mise en œuvre en région Rhône-Alpes d'une plateforme d'échanges de données dématérialisées entre les professionnels hospitaliers et libéraux (Zepra) devraient permettre d'améliorer la prise en charge médicamenteuse du patient ambulatoire [16].

\section{○ Non-conformité de la prescription médicale}

Les résultats attestent de l'importance de vérifier la conformité de la prescription ambulatoire avec le cadre réglementaire. Ce cadre conditionne le remboursement par l'Assurance Maladie, mais concourt également à sécuriser la prise en charge médicamenteuse du patient, en s'assurant par exemple qu'un traitement complexe requérant une spécialisation est prescrit par un médecin habilité, ou encore que le cadre du PUT est bien respecté (indication, posologie, suivi thérapeutique) pour éviter la iatrogénie médicamenteuse.

Ainsi, les problèmes de non-conformité aux référentiels et de contre-indications représentent la deuxième catégorie en terme de fréquence, dont près d'un tiers concerne un problème d'ATU non valide ou de PUT non respecté et un autre tiers un prescripteur non autorisé.

Les IP réalisées sont indispensables pour permettre la dispensation du traitement prescrit et ainsi éviter une rupture dans la continuité des traitements. 


\section{○ Problèmes d'observance}

Comme en officine de ville, les problèmes d'observance peuvent être détectés dans un suivi ambulatoire régulier comme c'est le cas pour les traitements chroniques (exemples : antirétroviraux, anticancéreux par voie orale, HTAP). Le prescripteur, informé en cas de défaut d'observance, pourra intégrer cette donnée dans la décision médicale (modification du traitement, consultation d'éducation thérapeutique). Le défaut d'observance a représenté 14 IP $(5,7 \%)$. Dans notre cas, il s'agit d'une méthode indirecte où seules les inobservances par défaut (intervalle trop long entre deux dispensations par rapport à la posologie prescrite) sont facilement décelables par l'équipe pharmaceutique et ont fait l'objet de mails aux prescripteurs. Par contre, il est difficile d'identifier les patients réguliers qui viennent chercher tous les mois leurs traitements sans les prendre (suite au décès d'un patient, sa famille nous a rapporté plus de 6 mois de traitement non utilisé).

\section{○ Problèmes de rupture dans la chaîne des soins}

Il s'agit le plus souvent de non-conformité à la liste de rétrocession ou de problèmes en lien avec la rupture d'un médicament en ville.

Les médicaments non rétrocédables représentent 3,2\% des interventions pour lesquelles il faut proposer au prescripteur un médicament de substitution disponible en ambulatoire, voire proposer un autre type de prise en charge pour le patient (hospitalisation à domicile lorsque cela est possible, hospitalisation de jour ou conventionnelle).

Dans 5,7 \% des problèmes rencontrés, il s'agit d'un médicament non prescrit après un transfert. Cet item recouvre par analogie les problèmes rencontrés lors de la rupture de médicaments en ville, pour laquelle les PUI sont largement impliquées dans la rétrocession de spécialités équivalentes importées. 
Pour exemple à la Pharmacie Centrale des HCL, ces situations de rupture ont représenté sur une période de 15 mois (du 01/01/12 au 30/04/13) $5 \%$ de l'activité [17] et généré des difficultés telles que discontinuité des traitements, difficulté de priorisation des patients (hôpital/ville) en cas de contingentement, risques d'erreurs médicamenteuses en cas de changement de schéma thérapeutique, de présentation ou de modalités de conservation du médicament de substitution importé par rapport au manquant.

Certaines IP sont récurrentes et nécessiteraient une information en amont auprès des prescripteurs, qui ignorent par exemple telle ou telle interaction médicamenteuse.

La multiplicité des prescripteurs rend une telle action difficile, la situation géographique également.

Le taux d'acceptation des IP par les cliniciens est élevé, ce qui montre la pertinence des interventions réalisées [3, 5-9].

Les médicaments impliqués reflètent en partie la spécialisation des consultations (maladies infectieuses, onco-hématologie, cardio-pneumologie, néonatologie, ...). Toute extrapolation avec l'hospitalisation induirait un biais.

Ce travail montre l'importance de l'analyse pharmaceutique des prescriptions ambulatoires et la nécessité de pouvoir disposer des mêmes éléments cliniques et biologiques que pour les patients hospitalisés (via le dossier médical commun informatisé) ainsi que de l'ensemble des prescriptions de ville. En effet, par exemple, lors des dispensations de teicoplanine pour des ostéites (traitement long), la difficulté réside dans l'obtention du taux résiduel réalisé en laboratoire d'analyse médicale de ville (avec la notion de sous-traitance entre laboratoires qui nous oblige parfois à contacter le laboratoire B qui effectue le dosage alors que le patient a été 
prélevé par le laboratoire A) avant de pouvoir dispenser le traitement. De plus, cette étude confirme la nécessité d'une coordination entre les prescripteurs HCL et hors HCL mais aussi entre les pharmaciens hospitaliers, officinaux et biologistes. La mise en cuvre d'une plateforme d'échanges de données dématérialisées entre les professionnels, intégrant notamment les données issues du dossier pharmaceutique du patient, devrait contribuer à l'amélioration de la prise en charge médicamenteuse du patient.

Dans un contexte de risque spécifique lié à l'organisation des parcours de soins, à l'origine d'incohérence potentielle entre les traitements, de points de rupture dans la continuité des traitements, la nécessité de mettre en place une conciliation médicamenteuse à l'entrée et à la sortie de l'hôpital, portant particulièrement sur les produits à risque, est capitale [18-21].

Par ailleurs, l'information délivrée lors de la sortie d'hospitalisation ou de consultation des patients sur les modalités d'accès aux traitements est encore parfois parcellaire et un effort particulier doit être apporté dans la communication auprès des unités de soins sur les outils d'information disponibles. Enfin, les pharmaciens référents des sites de rétrocession proposent également leur expertise dans l'organisation des sorties d'hospitalisation ou à destination de leurs confrères officinaux pour toute information relative au circuit et au bon usage du médicament. Les équipes sont d'ailleurs régulièrement sollicitées pour leur expertise, par des interlocuteurs très divers (patient, officinal, médecin, soignant, prestataire de santé à domicile), afin de répondre à des questions ne donnant pas lieu à une dispensation effective (exemple : rupture d'approvisionnement en ville, contingentement, réglementation, demande pour des patients étrangers), mais constituant autant de situations avec, à la clé, un impact clinique potentiel pour le patient (exemple : conformité aux référentiels, garantie de la continuité des soins) [22].

Cette étude présente plusieurs limites, celles liées à l'activité de rétrocession comme la spécialisation des différents sites, l'absence de DP et/ou d'accès aux données médicales mais 
aussi celles liées à l'étude comme la pluralité des codifications avec le facteur humain qui fait que chaque opérateur peut coder différemment selon son expérience et son ressenti, une grille de recueil non complètement efficiente malgré l'adaptation réalisée.

La mise à disposition par la SFPC de la grille dédiée à l'exercice officinal est attendue et permettra peut-être une meilleure adaptation à notre activité.

La base ACT-IP ${ }^{\circledR}$ ne permet pas l'enregistrement du niveau d'analyse. Par conséquent, nous n'avons pas codifié le niveau mis en œuvre pour aboutir aux interventions. Ainsi, nous ne pouvons pas conclure sur la répartition entre le nombre et le type d'IP en fonction du niveau.

\section{○ Perspectives}

Ce travail met en exergue l'importance des IP en rétrocession, ouvert à de nouvelles initiatives et à leurs évaluations par les prescripteurs en utilisant une échelle validée [23]. Des études sur la portée réelle des IP en termes de pharmaco-économie et de prévention de la morbidité iatrogène pourraient contribuer à valoriser les actions menées par les pharmaciens en rétrocession. 


\section{Conclusion}

L'interface des organisations ville-hôpital expose le patient à un risque accru d'erreurs médicamenteuses et également de rupture dans la continuité de ses traitements médicamenteux. L'analyse des prescriptions ambulatoires, en lien avec les prescriptions de ville, vise notamment à réduire ce risque, attesté par la typologie des principaux problèmes médicamenteux rencontrés dans ce travail.

Les résultats de cette étude montrent toute l'importance de l'analyse pharmaceutique pour prévenir la iatrogénie médicamenteuse dans ce contexte à risque particulier. Néanmoins, une limite se situe dans la multiplicité des circuits et des prescriptions potentielles (ville/hôpital) qui, en l'absence d'accès au dossier pharmaceutique partagé et/ou au dossier médical du patient, ne rend pas l'analyse exhaustive et donc totalement sécuritaire.

Le développement des liens entre les professionnels hospitaliers et libéraux devrait contribuer à optimiser les parcours de soins.

\section{Conflits d'intérêts : Aucun}




\section{Bibliographie :}

[1] : Malicki G., Bourleaud M., Cailleaux C., Conte E., Cote F., Duponchel A. et al. Guide de rétrocession du médicament, édition 2010, Observatoire du médicament, des dispositifs médicaux et de l'innovation thérapeutique de PACA \& Corse.

http://omedit.e-santepaca.fr/sites/omedit.e-

santepaca.fr/files/u4/Guide_Retrocession_ARS_PACA_Corse_2010_.pdf, consulté le $24 / 02 / 2014$

[2] : http://www.leem.org/article/remboursement-des-medicaments-taux-repartition-0, consulté le 24/02/2014.

[3] : Arques-Armoiry E., Cabelguenne D., Stamm C., Janoly-Dumenil A., Grosset-Grange I., Vantard N., et al. Problèmes médicamenteux les plus fréquemment détectés par l'analyse pharmaco-thérapeutique des prescriptions dans un centre hospitalier universitaire. Revue Med Interne 2010 ; 31 : 804-11.

[4] : Juste M. Recommandation de bonne pratique en pharmacie clinique. Analyse d'ordonnance et niveaux d'analyse pharmaceutique. Le Pharmacien Hospitalier et Clinicien, Volume 47, 2012, 293-295.

[5] : Bedouch P., Charpiat B., Conort O., Rose FX., Escofier L., Juste M., et al. Assessment of Clinical Pharmacists' Interventions in French Hospitals : Results of a Multicenter Study. Ann Pharmacother July 2008 42: 1095-103.

[6] : Charpiat B., Goutelle S., Schoeffler M., Aubrun F., Viale JP., Ducerf C.et al. Prescriptions analysis by clinical pharmacists in the post-operative period: a 4-year prospective study. Acta Anaesthesiol Scand, 2012 56: 1047-1051.

[7] : Caruba T., Colombet I., Gillaizeau F., Bruni V., Korb V., Prognon P. et al. Chronology of prescribing error during the hospital stay and prediction of pharmacist's alerts overriding: a prospective analysis. BMC Health Services Research 2010,10 : 13. 
[8] : Morice E., Gabriel-Bordenave C., Auclair V., Juste M., Roberge C. Evaluation of prescription checking practices and interventions by pharmacists in French hospitals. Le Pharmacien Hospitalier et Clinicien, Volume 46, 2011, e33-e48.

[9] : Estellat C., Colombet I., Vautier S., Huault-Quentel J., Durieux P., Sabatier B. Impact of pharmacy validation in a computerized physician order entry context. Int. J. Qual. Health. Care, $2007 ; 19: 317-325$.

[10] : Rose FX., Escofier L., Conort O., Charpiat B., Roubille R. Etude multicentrique de l'impact économique des interventions pharmaceutiques. Gestions Hospitalières 2009 ; $490: 549-54$

[11] : Conort O, Bedouch P, Juste M, Augereau L, Charpiat B, Roubille R, et al. Validation d'un outil de codification des interventions de pharmacie clinique. J.Pharm Clin, $2004 ; 23(3): 141-7$.

[12]: Bedouch P, Charpiat B, Roubille R, Juste M, Rose FX, Escofier L, et al. Documentation des interventions pharmaceutiques sur le site Internet de la Société française de pharmacie clinique : finalité, mode d'emploi et perspectives. J Pharm Clin $2007 ; 26(1): 40-3$.

[13] : Garcia C., Dessault J., Carpentier I., Locher F. Evaluation de l'adaptabilité de la fiche d'interventions pharmaceutiques proposée par la Société Française de Pharmacie Clinique à un service de rétrocession. Poster $n^{\circ}$ P_PCLIN_11, XVème congrès de la Société Française de Pharmacie Clinique, Grenoble, 5-7 février 2014.

[14] : George J., Majeed W., Mackenzie IS., Macdonald TM., Wei L. Association between cardiovascular events and sodium-containing effervescent, dispersible, and soluble drugs: nested case-control study. BMJ. 2013 Nov 26;347:f6954.

[15] : Bedouch P., Sylvoz N., Charpiat B., Juste M., Roubille R., Rose FX, et al. Trends in pharmacists' medication order review in French hospitals from 2006 to 2009: analysis of 
pharmacists' interventions from the Act-IP( ) website observatory. J Clin Pharm Ther. 2014 Oct 10. doi: 10.1111/jcpt.12214.

[16] : Spacagna H., Vandenbergh P., Garrigou-Granchamp M., Doerfler M., Durand T. Rhône-Alpes - La dématérialisation des échanges (ZEPRA) au service de la coordination ville-hôpital, première étape d'une meilleure coordination des parcours de santé. Eur Res Telemed $2014 ; 3(1): 39-40$.

[17] Hubault M., Carpentier I., Derain L., Locher F.,Meunier A. Ruptures d'approvisionnement des médicaments en ville : impact sur l'activité d'une pharmacie à usage intérieur ». Poster, Congrès Hopipharm, La Rochelle, 13-16 mai 2014.

[18] : Lebegue A.S., Le Bellec M. L., Truet S., Peronne E., Lemoine D. Conciliation médicamenteuse d'entrée et de sortie : optimisation et sécurisation du retour à domicile Pharm Hosp Clin 2013 ; 48(4) : 262-3.

[19] : Pérennes M., Carde A., Nicolas X., Dolz M., Bihannic R, Grimont P. et al. Conciliation médicamenteuse : une expérience innovante dans un service de médecine interne pour diminuer les erreurs d'anamnèse médicamenteuses. Presse Med $2012 ; 41(3)$ : e77-e86.

[20] : Dufour M., Philippe S., Bourdenet G., Borel C., Capet C., Jego A. et al. Conciliation médicamenteuse en médecine interne gériatrique : une collaboration efficace médecins - pharmaciens pour prévenir la iatrogénèse médicamenteuse. Étude prospective de 200 patients. Rev Med Interne 2013 ; 34(S2) : A117-A118.

[21] : Doerper S., Morice S., Piney D., Dony A., Baum T., Perrin F. et al. La conciliation des traitements médicamenteux : logigramme d'une démarche efficiente pour prévenir ou intercepter les erreurs médicamenteuses à l'admission du patient hospitalisé. Pharm Hosp Clin 2013 ; 48(3) : 153-60. 
[22] : Bart P., Garcia S., Dessault J., Carpentier I., Locher F. Expertise pharmaceutique hors dispensation au sein d'un service de rétrocession: Mise en place d'un outil informatique d'aide à la valorisation. Poster, Congrès Hopipharm, Lyon, 22-24 Mai 2013. [23]: Hatoum HT., Hutchinson RA, Witte KW, Newby GP. Evaluation of the contribution of clinical pharmacists: inpatient care and cost reduction. Drug Intell Clin Pharm. 1988; 22 (3) : 252-261. 
Tableau 1 : Nature des IP, des prescripteurs et devenir (fréquence)

Table 1 : Type of drug related problems, prescribers and acceptance rate (frequency)

\begin{tabular}{|c|c|c|c|c|}
\hline Nature des IP & $\mathrm{Nb}(\%)$ & $\begin{array}{l}\text { Acceptée } \\
(\%)\end{array}$ & $\begin{array}{l}\text { Refusée } \\
(\%)\end{array}$ & $\begin{array}{l}\text { Non } \\
\text { Renseignée } \\
(\%)\end{array}$ \\
\hline 1 - non-conformité aux référentiels et contre-indication (tout type) & $38(15,4)$ & 76,3 & 13,2 & 10,5 \\
\hline $1.1-$ non-conformité au livret & $8(3,2)$ & 100 & & \\
\hline 1.2 - non-conformité aux consensus & $10(4)$ & 60 & 40 & \\
\hline 1.3 - contre-indication & $2(0,8)$ & 100 & & \\
\hline 2- Indication non traitée & 0 & & & \\
\hline 2.1 - absence thérapeutique pour indication médicale validée & $5(2)$ & 60 & 20 & 20 \\
\hline 2.2 - médicament non prescrit après transfert & $14(5,7)$ & 92,9 & 7,1 & 0 \\
\hline $\begin{array}{l}\text { 2-3 Le patient n'a pas bénéficié d'une prescription de prophylaxie ou de } \\
\text { prémédication }\end{array}$ & 0 & & & \\
\hline 2-4 Un médicament synergique ou correcteur devrait être associé & 0 & & & \\
\hline 3- Sous-dosage & 0 & & & \\
\hline 3.1 - posologie infra-thérapeutique & $36(14,6)$ & 88,9 & 8,3 & 2,8 \\
\hline 3.2 - durée de traitement anormalement raccourcie & $1(0,4)$ & 100 & & \\
\hline 4- Surdosage & 0 & & & \\
\hline 4.1 - posologie supra-thérapeutique & $32(13)$ & 81,3 & 15,6 & 3,1 \\
\hline 4.2 - même principe actif prescrit plusieurs fois & $2(0,8)$ & 100 & & \\
\hline 5 - médicament non indiqué (tout type) & $3(1,2)$ & 100 & & \\
\hline 5.1 - médicament prescrit sans indication justifiée & $1(0,4)$ & 100 & & \\
\hline $\begin{array}{c}5.2 \text { - médicament prescrit sur une durée trop longue sans risque de } \\
\text { surdosage }\end{array}$ & $3(1,2)$ & 66,7 & 33,3 & \\
\hline $\begin{array}{l}\text { 5-3 Prescriptions de deux médicaments à principe actif différent mais } \\
\text { appartenant à la même classe thérapeutique créant une redondance } \\
\text { pharmacologique }\end{array}$ & 0 & & & \\
\hline 6- Interaction & 0 & & & \\
\hline 6.1 - à prendre en compte & $3(1,2)$ & 100 & & \\
\hline 6.2 - Précaution d'emploi & $1(0,4)$ & 100 & & \\
\hline 6.3 - association déconseillée & $10(4)$ & 80 & 10 & 10 \\
\hline $6.4-$ contre-indication & $17(6,9)$ & 88,2 & 5,9 & 5,9 \\
\hline 6-5 non publiée (qq références bibliographiques) & 0 & & & \\
\hline 7 - effet indésirable & $1(0,4)$ & 100 & & \\
\hline 8 - voie et/ou administration inappropriée (tout type) & $2(0,8)$ & 100 & & \\
\hline 8-1 Autre voie plus efficace, ou moins coûteuse à efficacité équivalente & 0 & & & \\
\hline 8.2 - méthode d'administration non adéquate & $8(3,2)$ & 62,5 & 12,5 & 25 \\
\hline 8.3 - mauvais choix de galénique & $5(2)$ & 100 & & \\
\hline 8.4 - libellé incomplet & $11(4,5)$ & 100 & & \\
\hline 8.5 - plan de prise non optimal & $7(2,8)$ & 71,4 & 28,6 & \\
\hline 9 - traitement non reçu (tout type) & $4(1,6)$ & 100 & & \\
\hline 9.1 - incompatibilité physico-chimique entre médicaments injectables & $1(0,4)$ & & & 100 \\
\hline 9.2 - problème d'observance & $14(5,7)$ & 42,9 & & 57,1 \\
\hline 10 - monitorage à suivre & $8(3,2)$ & 100 & & \\
\hline Total & 247 & $\mathbf{8 1 , 8}$ & 10,1 & $\mathbf{8 , 1}$ \\
\hline Nature des prescripteurs & $\mathrm{Nb}(\%)$ & $\begin{array}{l}\text { Acceptée } \\
(\%)\end{array}$ & $\begin{array}{l}\text { Refusée } \\
(\%)\end{array}$ & $\begin{array}{c}\text { Non } \\
\text { Renseignée } \\
(\%)\end{array}$ \\
\hline Interne & $38(15,4)$ & $35(92,1)$ & $3(7,9)$ & \\
\hline Senior & $209(84,6)$ & $167(79,9)$ & $22(10,5)$ & $20(9,6)$ \\
\hline
\end{tabular}


Tableau 2 : Interventions proposées et mode de transmission (fréquence)

Table 2 : Interventions type and communication mode (frequency)

\begin{tabular}{|cc|}
\hline Interventions proposées & Nb (\%) \\
\hline 1 - Ajout (prescription nouvelle) & $\mathbf{5 1}(\mathbf{2 0 , 6})$ \\
2 - Arrêt & $18(7,3)$ \\
3 - Substitution/Échange & $29(11,7)$ \\
4 - Choix de la voie d'administration & $2(0,8)$ \\
5 - Suivi thérapeutique & $\mathbf{3 7}(\mathbf{1 5})$ \\
6 - Optimisation des modalités & $\mathbf{3 8}(\mathbf{1 5 , 4 )}$ \\
d'administration & $\mathbf{7 2}(\mathbf{2 9 , 1})$ \\
7 - Adaptation posologique & $\mathbf{N b}(\mathbf{\%})$ \\
\hline Mode de transmission & $3(1,2)$ \\
Oralement & $\mathbf{2 0 6}(\mathbf{8 3 , 4})$ \\
Appel téléphonique & $19(7,7)$ \\
Appel téléphonique et e-mail & $19(7,7)$ \\
E-mail &
\end{tabular}

Tableau 3 : Origine des non-conformités (fréquence)

Table 3 : Origin of unconformities (frequency)

\begin{tabular}{|cc|}
\hline Origine des non-conformités & Nb $(\boldsymbol{\%})$ \\
\hline Prescripteur non autorisé/habilité & $\mathbf{1 6}(\mathbf{4 2 , 1 )}$ \\
Défaut d'ATU & $11(28,9)$ \\
Non-respect du PUT & $6(15,8)$ \\
Support non-conforme & $5(13,2)$ \\
\hline
\end{tabular}


Tableau 4 : Classe ATC des médicaments concernés et devenir (fréquence).

Table 4 : ATC drugs groups causing drug related problems and acceptance rate (frequency)

\begin{tabular}{|c|c|c|c|c|}
\hline Classe ATC des médicaments & $\mathrm{Nb}(\%)$ & $\begin{array}{l}\text { Acceptée } \\
(\%)\end{array}$ & $\begin{array}{l}\text { Refusée } \\
(\%)\end{array}$ & $\begin{array}{c}\text { Non } \\
\text { Renseignée } \\
(\%)\end{array}$ \\
\hline A : Système digestif et métabolisme & $8(3,2)$ & 62,5 & 37,5 & \\
\hline B : Sang et organes hématopoiétiques & $14(5,7)$ & 78,6 & 14,3 & 7,1 \\
\hline C : Système cardio-vasculaire & $10(4)$ & 90 & & 10 \\
\hline D : Dermatologie & 0 & & & \\
\hline G : Système génito-urinaire et hormones sexuelles & $11(4,5)$ & 90,9 & 9,1 & \\
\hline $\begin{array}{c}\mathrm{H} \text { : Préparations systémiques hormonales à l'exclusion des hormones } \\
\text { sexuelles et des insulines }\end{array}$ & $15(6,1)$ & 93,3 & 6,7 & \\
\hline $\mathrm{J}:$ Anti-infectieux (usage systémique) & $108(43,7)$ & 78,7 & 10,2 & 11,1 \\
\hline $\mathrm{L}$ : Antinéoplasiques et agents immunomodulants & $43(17,4)$ & 90,7 & 4,7 & 4,7 \\
\hline M : Système musculo-squelettique & $1(0,4)$ & 100 & & \\
\hline $\mathrm{N}$ : Système nerveux & $16(6,5)$ & 81,3 & 12,5 & 6,3 \\
\hline $\mathrm{P}$ : Produits antiparasitaires, insecticides et répellants & $6(2,4)$ & 83,3 & 16,7 & \\
\hline $\mathrm{R}:$ Système respiratoire & 0 & & & \\
\hline S : Organes sensoriels & $5(2)$ & 40 & 40 & 20 \\
\hline V : Divers & $3(1,2)$ & 100 & & \\
\hline X : Diététiques (hors ATC) & $2(0,8)$ & 50 & & 50 \\
\hline Z: Non codé (hors ATC) & $5(2)$ & 80 & & 20 \\
\hline Sous-classe ATC des médicaments & $\begin{array}{c}\text { Nb } \\
(\% / \text { classe })\end{array}$ & \multicolumn{3}{|c|}{ Exemples } \\
\hline B02 : Antihémorragiques & $7(50)$ & \multicolumn{3}{|c|}{ Facteurs antihémophiliques } \\
\hline B03 : Préparations Antianémiques & $4(28,6)$ & \multicolumn{3}{|c|}{ Spécialité de fer injectable } \\
\hline G04 : Médicaments urologiques & $11(100)$ & \multicolumn{3}{|c|}{ Sildénafil pour l'HTAP } \\
\hline H01 : Hormones hypophysaires, hypothalamiques et analogues & $2(13,3)$ & \multicolumn{3}{|c|}{ Thyrotropine alfa } \\
\hline H02 : Corticoides à usages systémiques & $11(73,3)$ & \multicolumn{3}{|c|}{ Dexamathésone sous ATU } \\
\hline J01 : Antibactériens à usage systémique & $47(43,5)$ & \multicolumn{3}{|c|}{$\begin{array}{l}\text { Fludrocortisone et propylthiouracile } \\
\text { suite à la rupture en ville. } \\
\text { Problèmes d'antibiogramme, de } \\
\text { posologie, de contre-indication entre } \\
\text { linézolide et tramadol }\end{array}$} \\
\hline J02 : Antimycosiques à usage systémique & $17(15,7)$ & \multicolumn{3}{|c|}{$\begin{array}{l}\text { Problèmes d'interactions avec CYP, de } \\
\text { posologies ou de mode de prise }\end{array}$} \\
\hline J05 : Antiviraux à usage systémique & $36(33,3)$ & \multicolumn{3}{|c|}{$\begin{array}{l}\text { Contre-indication, problème } \\
\text { d'observance }\end{array}$} \\
\hline L01 : Antinéoplasiques & $28(65,1)$ & \multicolumn{3}{|c|}{ Thalidomide, Témézolomide } \\
\hline L04 : Immunosuppresseurs & $10(23,3)$ & \multicolumn{3}{|c|}{ Lénalidomide } \\
\hline N03 : Antiépileptiques & $7(43,8)$ & \multicolumn{3}{|c|}{ Phenytoïne suite à rupture ville } \\
\hline N06 : Psychoanaleptiques & $5(31,3)$ & \multicolumn{3}{|c|}{ Trazodone } \\
\hline
\end{tabular}

